\title{
Contribution of Lateral Interbody Fusion in Staged Correction of Adult Degenerative Scoliosis
}

\author{
Seung Won Choi, M.D., Ph.D., ${ }^{1}$ Christopher Ames, M.D., ${ }^{2}$ Sigurd Berven, M.D., ${ }^{3}$ Dean Chou, M.D., ${ }^{2}$ Bobby Tay, M.D., ${ }^{3}$ \\ Vedat Deviren, M.D. ${ }^{3}$ \\ Department of Neurosurgery, Chungnam National University Hospital, Daejeon, Korea \\ Department of Neurosurgery, ${ }^{2}$ University of California San Francisco, San Francisco, CA, USA \\ Department of Orthopaedic Surgery, ${ }^{3}$ University of California San Francisco, San Francisco, CA, USA
}

Objective : Lateral interbody fusion (LIF) is attractive as a less invasive technique to address anterior spinal pathology in the treatment of adult spinal deformity. Its own uses and benefits in treatment of adult degenerative scoliosis are undefined. To investigate the radiographic and clinical outcomes of LIF, and staged LIF and posterior spinal fusion (PSF) for the treatment of adult degenerative scoliosis patients, we analyzed radiographic and clinical outcomes of adult degenerative scoliosis patients who underwent LIF and posterior spinal fusion.

Methods : Forty consecutive adult degenerative scoliosis patients who underwent LIF followed by staged PSF at a single institution were retrospectively reviewed. Long-standing 36 " anterior-posterior and lateral radiographs were taken preoperatively, at inter-stage, 3 months, 1 year, and 2 years after surgery were reviewed. Outcomes were assessed through the visual analogue scale (VAS), 36-Item Short Form Health Survey (SF-36), and Oswestry Disability Index (ODI).

Results : Forty patients with a mean age of 66.3 (range, 49-79) met inclusion criteria. A mean of 3.8 levels (range, 2-5) were fused using LIF, while a mean of 9.0 levels (range, 3-16) were fused during the posterior approach. The mean time between stages was 1.4 days (range, 1-6). The mean follow-up was 19.6 months. Lumbar lordosis was significantly restored from $36.4^{\circ}$ preoperatively up to $48.9^{\circ}$ (71.4\% of total correction) after LIF and $53.9^{\circ}$ after PSF. Lumbar coronal Cobb was prominently improved from $38.6^{\circ}$ preoperatively to $24.1^{\circ}\left(55.8 \%\right.$ of total correction) after LIF, $12.6^{\circ}$ after PSF respectively. The mean pelvic incidence-lumbar lordosis mismatch was markedly improved from $22.2^{\circ}$ preoperatively to $8.1^{\circ}\left(86.5 \%\right.$ of total correction) after LIF, $5.9^{\circ}$ after PSF. Correction of coronal imbalance and sagittal vertebral axis did not reach significance. The rate of perioperative complication was $37.5 \%$. Five patients underwent revision surgery due to wound infection. No major perioperative medical complications occurred. At last followup, there were significant improvements in VAS, SF-36 Physical Component Summary and ODI scores.

Conclusion : LIF provides significant corrections in the coronal and sagittal plane in the patients with adult degenerative scoliosis. However, LIF combined with staged PSF provides more excellent radiographic and clinical outcomes, with reduced perioperative risk in the treatment of adult degenerative scoliosis.

Key Words : Spine · Deformity · Fusion · Correction.

-Received : October 23, 2017 •Revised : December 3, 2017 •Accepted : December 17, 2017

- Address for reprints : Vedat Deviren, M.D.

Department of Orthopaedic Surgery, University of California San Francisco, 400 Parnassus Avenue, San Francisco, CA 94143-0332, USA

Tel : +1-866-817-7463, Fax : +1-415-353-4047, E-mail : Vedat.Deviren@ucsf.edu

This is an Open Access article distributed under the terms of the Creative Commons Attribution Non-Commercial License (http://creativecommons.org/licenses/by-nc/4.0) which permits unrestricted non-commercial use, distribution, and reproduction in any medium, provided the original work is properly cited. 


\section{INTRODUCTION}

Current surgical treatment options for adult degenerative scoliosis include anterior-only, posterior-only and combined anterior and posterior procedures ${ }^{3,7-11,14,18-26)}$. A primary goal of any surgical intervention is the restoration of the coronal and sagittal balance, as this is directly correlated with patient outcomes. Recent attention has been given to the lateral interbody fusion (LIF) technique for addressing anterior spinal pathology. Furthermore LIF has been combined with posterior spinal fusion (PSF) in staged correction of spinal deformity. With the frequency of spinal deformity procedures increasing as the population ages, it is important to understand the merits of LIF combined with staged PSF for addressing adult spinal deformity ${ }^{5)}$.

The first aim of this study was to assess the magnitude of radiographic changes following staged LIF and PSF. For this task, we reviewed radiographs preoperatively, following LIF and before PSF (inter-stage), after PSF and at the most recent clinical follow up. We analyzed the degree of radiographic change between these time periods in order to determine each procedures contribution to the final surgical correction. A second aim of this study was to analyze the functional outcomes, perioperative and postoperative complications of patients undergoing staged LIF and PSF procedures.

\section{MATERIALS AND METHODS}

\section{Study design and patient selection}

Consecutive patients with adult degenerative scoliosis who underwent staged correction using LIF and PSF at a tertiary care spine referral center between 2008 and 2013 were enrolled. The diagnosis of adult degenerative scoliosis included at least one of the following : coronal Cobb angle greater than 20 degrees, sagittal imbalance greater than $5 \mathrm{~cm}$, coronal imbalance greater than $5 \mathrm{~cm}$, thoracic kyphosis greater than 60 degrees, thoracolumbar kyphosis greater than 20 degrees, or lumbar lordosis less than 20 degrees. Prior to surgery, all patients failed a trial of conservative treatment for at least 6 months. Patients were enrolled prospectively, and medical records were reviewed retrospectively up to the most recent clinical evaluation.

\section{Operative sequences}

\section{First stage}

LIF was performed on the indicated lumbar levels using a lateral trans-psoas approach with neuromonitoring. For thoracic and upper lumbar level LIF, a lateral transthoracic or thoracoabdominal approach was performed. When the patient had severe bridging osteophytes, osteotomies were performed on the contralateral side with a Cobb. After the discectomy, a lordotic interbody cage (Nuvasive, San Diego, CA, USA) packed with allogenic bone graft substitute (Osteocel Nuvasive, San Diego, CA, USA) containing live mesenchymal stem cells was inserted to the intervertebral disc spaces. Interbody cages ranged from length 45-60 $\mathrm{mm}$, height 8-12 $\mathrm{mm}$, width 18-22 mm, and lordosis angle 10-30 degrees. The first stage was followed by an inter-stage of between 1-6 days, wherein the patient underwent standing long cassette 36 " anterior-posterior and lateral radiographs, and were adequately resuscitated for the second procedure.

\section{Second stage}

Posterior decompressive laminectomies, Smith-Petersen osteotomies, placement of pedicle screws, laminar hooks and iliac screws were performed. Fluoroscopy or an intra-operative computed tomography scan was used for confirmation of adequate placement of instrumentation before completion of the operation. For the patients who were not able to undergo LIF at L5-S1 due to the iliac crest, but required additional fusion at L5-S1, anterior lumbar interbody fusion (ALIF) or transforaminal interbody fusion (TLIF) was performed. We tried to correct the deformities more to obtain the optimal angles by rod contouring and compression after Smith-Peterson osteotomy during 2nd surgery. We used cobalt chromium rods and performed posterolateral fusion with mixture of allogenic bone chips and harvested autologous bone chips.

\section{Data collection}

\section{Radiographic data}

Standing long cassette 36" anterior-posterior and lateral radiographs taken preoperatively, at inter-stage, 3 months, 1 year, and 2 years after surgery were reviewed. Lumbar lordosis (LL), pelvic tilt (PT), pelvic incidence (PI), coronal Cobb angle, coronal imbalance, sagittal vertical axis (SVA) were mea- 
sured using Surgimap Spine radiographic mapping software (version 1.2.1.86; Nemaris, Inc., New York, NY, USA). LL angle was measured from the superior endplate of L1 to the superior endplate of S1. PT was measured as the angle between the lines connecting the midpoint of the sacral plate to the bicoxofemoral axis and the vertical plane. PI was calculated from the angle between a perpendicular line to the sacral plate and the line connecting the midpoint of the sacral plate to the bicoxofemoral axis.

PI-LL mismatch was also determined. Coronal Cobb angle was determined as the angle formed by the superior end plate of the most angulated superior vertebral body and the inferior end plate of the most angulated interior vertebral body. Coronal imbalance was determined by measuring the distance between $\mathrm{C7}$ plumb line which is a vertical line drawn down from the mid-portion of the $\mathrm{C} 7$ vertebral body and central sacral vertical line which is a perpendicular line drawn up through the center of the sacrum on anteroposterior radiographs. SVA was measured as the horizontal distance between the C7 plumb line and the posterosuperior corner of the sacrum.

\section{Clinical data}

Functional outcomes were assessed by evaluating visual analogue scale (VAS) for back pain and leg pain, Oswestry Disability Index (ODI), 36-Item Short Form Health Survey mental component summary (SF-36 MCS), and 36-Item Short Form Health Survey physical component summary (SF-36 PCS) preoperatively, and at 3, 12, and 24 months after surgery. Perioperative and postoperative complications were also investigated.

\section{Statistical analysis}

Radiographic parameters and functional outcome variables were compared with their corresponding values using a paired t test. All statistical tests were two-sided and $p$-values less than 0.05 were considered to be significant statistically. Data with normal distribution has been assigned a mean and standard deviation. SPSS version 21 (IBM, New York, NY, USA) was used for statistical analysis.

\section{RESULTS}

\section{Demographic and operative data}

Forty consecutive patients with adult degenerative scoliosis were enrolled. The mean age at surgery was 66.3 years (range, 40-79; standard deviation, \pm 7.7 ) and $87.5 \%$ were female. Twelve patients (30\%) had a history of prior back surgery.

Interbody fusion was performed at 178 levels (mean level per patient, 4.5; range, 2-6). This included 151 LIF (mean level per patient, 3.8; range, 2-5) in the thoracolumbar spine and 25 TLIF and two ALIF at L5-S1 interspace. Three hundred fiftynine vertebral segments were fused posteriorly (mean level per patient, 9; range, 3-16). In 38 patients (95\%) iliac screws were placed, and no pedicle subtraction osteotomies were performed in any patients. Operative data is summarized in Table 1. Mean time of the inter-stage was 1.4 days (range, 1-6) and mean hospital stay was 8.6 days (range, 5-22). Mean follow-up duration after LIF was 22.2 months (range, 7-54).

\section{Radiographic results}

Table 2 summarizes the radiographic results. Table 3 shows respective correction rate and contribution rate of LIF and PSF to total deformity. Radiographic measurements of preoperative, inter-stage, postoperative and last clinical follow up including long-standing 36" films were adequately obtained in 34 patients (85\%) (Fig. 1). In six patients, there were some radiographs that were unavailable. Mean duration until last radiographic follow-up was 17.4 months.

The mean preoperative LL was $36.4^{\circ} \pm 13.7^{\circ}$ which was significantly changed to $48.9^{\circ} \pm 12.8^{\circ}$ after LIF $(71.4 \%$ of total staged correction) and $53.9^{\circ} \pm 12.6^{\circ}$ after PSF $(28.6 \%$ of total

Table 1. Operative data

\begin{tabular}{lc}
\hline & Value \\
\hline Lateral interbody fusion & 151 \\
Total levels & 40 \\
Patients & $3.8 \pm 0.7$ \\
Levels per patient & \\
\hline Fusion at L5-S1 & 25 \\
TLIF & 2 \\
ALIF & $4.5 \pm 1.0$ \\
Levels of interbody fusion per patient & $9.0 \pm 3.9$ \\
Levels of posterior spinal fusion per patient & $38(95)$ \\
Fusion to pelvis & $1.4 \pm 1.2$ \\
Interval between stages
\end{tabular}

Values are presented as mean \pm standard deviation or number (\%). TILF : transforaminal lumbar interbody fusion, ALIF : anterior lumbar interbody fusion 
Table 2. Radiographic results

\begin{tabular}{|c|c|c|c|c|c|c|c|}
\hline & \multirow{2}{*}{$\begin{array}{c}\text { Preoperative } \\
\text { Mean } \pm \text { SD }\end{array}$} & \multicolumn{2}{|c|}{ After LIF } & \multicolumn{2}{|c|}{ After PSF* } & \multicolumn{2}{|c|}{ Last follow-up ${ }^{\dagger}$} \\
\hline & & Mean \pm SD & $p$-value & Mean \pm SD & $p$-value & Mean \pm SD & $p$-value \\
\hline Lumbar lordosis $\left({ }^{\circ}\right)$ & $36.4 \pm 13.7$ & $48.9 \pm 12.8$ & $<0.001$ & $53.9 \pm 12.6$ & $<0.001$ & $56.1 \pm 11.5$ & $<0.001$ \\
\hline Pelvic tilt ( $\left.{ }^{\circ}\right)$ & $23.0 \pm 9.7$ & $15.6 \pm 9.2$ & $<0.001$ & $20.6 \pm 9.1$ & 0.12 & $21.2 \pm 8.2$ & 0.255 \\
\hline $\mathrm{PI}-\mathrm{LL}$ mismatch $\left({ }^{\circ}\right)$ & $22.2 \pm 15.9$ & $8.1 \pm 13.9$ & $<0.001$ & $5.9 \pm 12.7$ & $<0.001$ & $4.0 \pm 13.4$ & $<0.001$ \\
\hline Thoracic coronal Cobb () & $20.8 \pm 13.0$ & $16.0 \pm 14.7$ & $<0.001$ & $9.2 \pm 10.2$ & $<0.001$ & $9.3 \pm 9.7$ & $<0.001$ \\
\hline Lumbar coronal Cobb () & $38.6 \pm 19.5$ & $24.1 \pm 16.6$ & $<0.001$ & $12.6 \pm 8.2$ & $<0.001$ & $13.5 \pm 9.0$ & $<0.001$ \\
\hline Coronal imbalance (mm) & $19.2 \pm 27.3$ & $26.6 \pm 32.8$ & 0.222 & $15.3 \pm 18.1$ & 0.384 & $15.7 \pm 22.8$ & 0.428 \\
\hline Sagittal vertical axis (mm) & $61.7 \pm 49.5$ & $58.2 \pm 63.6$ & 0.88 & $42.2 \pm 44.3$ & 0.039 & $42.0 \pm 49.5$ & 0.058 \\
\hline
\end{tabular}

p-values compared to preoperative values. * Three months after posterior spinal fusion. ${ }^{\dagger}$ Mean duration until last radiographic follow-up was 17.4 months. LIF : lateral interbody fusion, PSF : posterior spinal fusion, PI-LL : pelvic incidence-lumbar lordosis

Table 3. Rate of correction and contribution

\begin{tabular}{|c|c|c|c|c|c|}
\hline & \multicolumn{2}{|c|}{ LIF (\%) } & \multicolumn{2}{|c|}{ PSF (\%) } & \multirow{2}{*}{$\begin{array}{c}\text { Total (\%) } \\
\text { Correction* }\end{array}$} \\
\hline & Correction* & Contribution $^{\dagger}$ & Correction* & Contribution $^{\dagger}$ & \\
\hline Lumbar lordosis & 34.3 & 71.3 & 13.8 & 28.7 & 48.1 \\
\hline PI-LL mismatch & 63.5 & 86.8 & 9.6 & 13.2 & 73.2 \\
\hline Thoracic coronal Cobb & 23 & 41.4 & 32.6 & 58.6 & 55.6 \\
\hline Lumbar coronal Cobb & 37.6 & 55.9 & 29.7 & 44.1 & 67.3 \\
\hline Sagittal vertical axis & 5.7 & 18 & 25.9 & 82 & 31.6 \\
\hline
\end{tabular}

*Values indicate correction rate to preoperative measurements. ${ }^{\top}$ Values indicate contribution rate to final postoperative measurements. LIF : lateral interbody fusion, PSF : posterior spinal fusion, PI-LL : pelvic incidence-lumbar lordosis
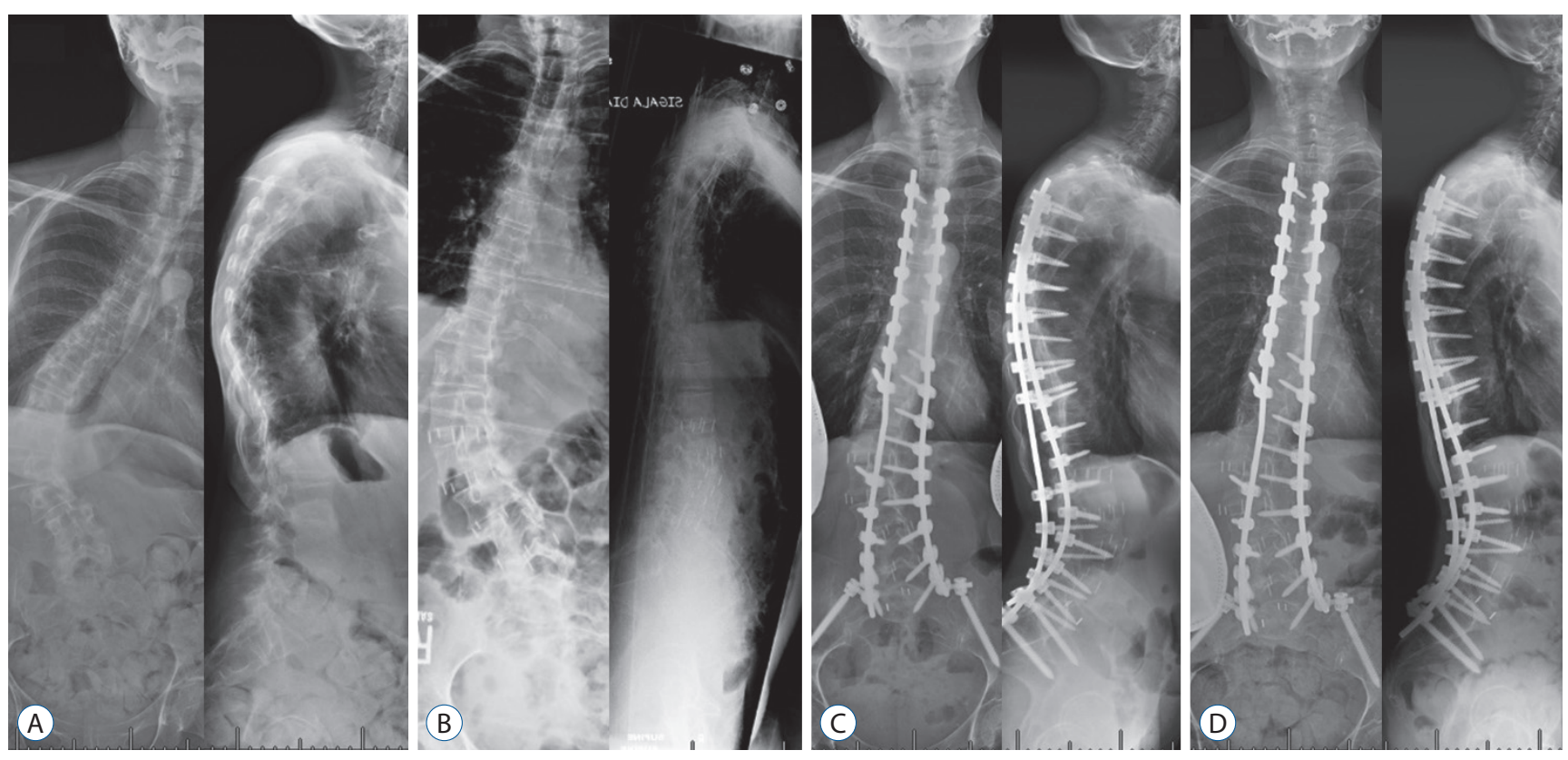

Fig. 1. In a 60-year-old female, preoperative coronal Cobb angle of $69^{\circ}$ and lumbar lordosis of $46^{\circ}$ (A) were corrected to $47^{\circ}$ and $60^{\circ}$ after lateral interbody fusion (B) and $26^{\circ}$ and $58^{\circ}$ after posterior spinal fusion (C). The angles measured $27^{\circ}$ and $54^{\circ}$ respectively at 2 years later (D). 
staged correction). LL continued to increase to $56.1^{\circ} \pm 11.5^{\circ}$ at time of last follow-up. All values were significantly different from pre-operative lumbar lordosis $(p<0.001)$.

PT decreased significantly from the preoperative mean of $23.0^{\circ} \pm 9.7^{\circ}$ to $15.6^{\circ} \pm 9.2^{\circ}$ at the interstage $(p<0.001)$. However PT was shown to return to near pre-operative values on imaging following PSF and the most recent clinical follow up. The mean PI-LL mismatch was significantly improved from $22.2^{\circ} \pm 15.9^{\circ}$ preoperatively to $8.1^{\circ} \pm 13.9^{\circ}$ after LIF ( $86.5 \%$ of total staged correction), and $5.9^{\circ} \pm 12.7^{\circ}$ after PSF ( $13.5 \%$ of total staged correction) and $4.0^{\circ} \pm 13.5^{\circ}$ at last follow-up. All values were significantly different than preoperative PI-LL mismatch $(p<0.001)$.

The thoracic major coronal Cobb angle was $20.8^{\circ} \pm 13.0^{\circ}$ preoperatively, $16.0^{\circ} \pm 14.7^{\circ}$ after LIF ( $41.4 \%$ of total staged correction), $9.2^{\circ} \pm 10.2^{\circ}$ after PSF (58.6\% of total staged correction) and $9.3^{\circ} \pm 9.7^{\circ}$ at last follow up. The mean lumbar coronal Cobb was $38.6^{\circ} \pm 19.5^{\circ}$ preoperatively which improved to $24.1^{\circ}$ $\pm 16.6^{\circ}$ after LIF (55.4\% of total staged correction), $12.6^{\circ} \pm 8.2^{\circ}$ after PSF (44.6\% of total staged correction) and $13.5^{\circ} \pm 9.0^{\circ}$ at last follow-up. All values were significantly different than preoperative thoracic and lumbar coronal Cobb angles $(p<0.001)$.

The coronal imbalance increased from $19.2 \pm 27.3 \mathrm{~mm}$ to $26.6 \pm 32.8 \mathrm{~mm}$ after LIF. However, following PSF coronal imbalance was $15.3 \pm 18.1 \mathrm{~mm}$, and $15.7 \pm 22.8 \mathrm{~mm}$ at last followup. None of these values were significantly different than the pre-operative measurement. SVA decreased from $61.7 \pm 49.5$ $\mathrm{mm}$ preoperatively to $58.2 \pm 63.6 \mathrm{~mm}$ after LIF ( $p=0.88 \mathrm{com}$ pared with pre-operative) (17.9\% of total staged correction), to $44.2 \pm 44.3 \mathrm{~mm}$ ( $p=0.039$ compared to pre-operative), and 42.0 $\pm 49.5 \mathrm{~mm}(p=0.058)$ at final follow up.

\section{Clinical results}

The mean VAS for back pain decreased significantly from $7.3 \pm 2.3$ pre-operatively to $3.4 \pm 2.2$ at 3 -month follow-up $(p<0.001)$ and $4.4 \pm 2.9$ at last follow-up $(p<0.001)$. The mean VAS for leg pain decreased significantly from $5.4 \pm 3.7$ preoperatively to $2.4 \pm 2.8$ at 3 -month follow-up $(p=0.005)$ and $2.9 \pm 3.1$ at last follow-up $(p=0.02)$. The mean ODI significantly increased from $49.6 \pm 16.0$ preoperatively to $39.1 \pm 17.6$ at 3 -month follow-up $(p=0.001)$ and 33.9 \pm 18.7 at last follow-up $(p<0.001)$. The mean SF-36 PCS significantly increased from 26.7 \pm 9.2 preoperatively to $33.7 \pm 8.7$ at 3 -month follow-up ( $p=0.003$ ) and $34.5 \pm 10.0$ at last follow-up ( $p=0.001$ ). The SF-36 MCS did not show significant changes postoperatively.

\section{Complications}

Perioperative complications in this study were categorized as major or minor and surgical or medical, based on the report of Isaacs et al. ${ }^{15)}$ Fifteen patients (37.5\%) had perioperative complications. Among them, five patients had a major complication (12.5\%). Each major complication was an infection, which required additional surgery. There were no major medical complications. All perioperative complications are described in Table 4.

\section{DISCUSSION}

Surgical strategies and techniques for decompression of neural elements and correction of adult spinal deformity are generally divided into anterior, posterior and combined procedures. Recently, attention has been given to the lateral transpsoas approach to the anterior lumbar spinal column ${ }^{222}$. This technique decreases the risks associated with an anterior approach such as bowel injuries, vascular injuries, ileus and retrograde ejaculation. Additionally, LIF provides the ability to release, reconstruct and fuse the anterior column, and indirectly decompress the neural elements by disc distraction and spinal alignment ${ }^{2,23)}$. For these reasons, LIF combined with PSF has become a popular procedure for the treatment of adult degenerative scoliosis. To our knowledge, there are no other studies that demonstrate

Table 4. Perioperative complications

\begin{tabular}{lc}
\hline & Value \\
\hline Major & 5 \\
Medical & 0 \\
Surgical & 5 \\
Wound Infection & 5 \\
\hline Minor & 10 \\
Medical & 6 \\
Delirium & 2 \\
Atelectasis & 1 \\
Atrial fibrillation & 1 \\
Tachicardia & 1 \\
Electroly imbalance & 1 \\
Surgical & 4 \\
Ileus & 2 \\
Dural tear & 1 \\
Diaphragmatic tear & 1 \\
\hline
\end{tabular}


the isolated radiographic effect of LIF, LIF's contribution to the total correction of staged LIF-PSF, perioperative complications, and long term radiographic and clinical follow up.

In the present study, LL was restored from $36.4^{\circ}$ to $53.9^{\circ}$ after staged correction, with LIF contributing $71.4 \%$ of the total correction. Prior research has suggested that LIF does not improve global lumbar lordosis ${ }^{1,6,8,11,12,16,17,23,27,28)}$, however this study demonstrated the contrary. This result is thought to be due to the number of LIF levels performed. We performed LIF in a greater number of levels (mean, 3.8 levels per patient) than any other report. In contrast, Tormenti et al. ${ }^{28)}$ performed a mean of 2.8 levels, Acosta et al. ${ }^{1)}$ performed a mean of $1.8 \mathrm{lev}-$ els and Johnson et al. ${ }^{16)}$ performed a mean of 1.1 levels of LIF.

It is additionally important to assess the correction of coronal curvature with varying surgical techniques. Yadla et al. ${ }^{29)} \mathrm{s}$ research on over 2000 adults with degenerative scoliosis reported the average postoperative correction of coronal deformity as $40.7 \%$. Phillips et al. ${ }^{23)}$ reported 35\% correction of coronal Cobb angle in adult degenerative scoliosis treated with XLIF. In this study, coronal Cobb angles were significantly restored in both the thoracic and lumbar region after staged correction.

In this study, PT decreased significantly from the preoperative mean of $23.0^{\circ} \pm 9.7^{\circ}$ to $15.6^{\circ} \pm 9.2^{\circ}$ at the interstage, then however it was increased to $20.6^{\circ} \pm 9.1^{\circ}$ at 3 months after PSF and $21.2^{\circ} \pm 8.2^{\circ}$ at the last follow-up. The mean interval between the first and second operation was 1.4 days. So, the patients usually took the standing whole spine radiographs at the day after the first surgery. At this time, the surgical pain was still severe, and the head and upper body were forwarded which resulted in temporarily anterior tilting of the pelvis when standing. We thought this is the reason for excessive decrease of PT after LIF.

Few studies about the influence of LIF on global coronal or sagittal imbalance have been reported in the literature ${ }^{1,6,27)}$. These studies did not demonstrate a significant effect of LIF on sagittal vertebral axis and coronal imbalance. This study agrees with prior literature, wherein LIF did not significantly alter sagittal vertebral axis. However, following PSF there was significant restoration of sagittal axis $(p=0.039)$. This restoration trended towards significance at final follow up $(p=0.058)$. Coronal imbalance was not affected by LIF and PSF in this study.

In spite of variable statistical significance, the clinical outcomes of LIF in the treatment of degenerative scoliosis have been reported with good results ${ }^{4,11,13,15,17,20,23,27)}$. In our study,
VAS for back pain, VAS for leg pain, ODI and SF-36 PCS at last follow-up were significantly improved following staged correction. However, SF-36 MCS did not reach significance statistically. These results were consistent with prior research by Tempel et al. ${ }^{27)}$.

Isaacs et al. ${ }^{15)}$ reported a major complication rate of $12.1 \%$ and overall complication rate of $24.3 \%$ following LIF in the treatment of adult scoliosis. Additionally, in cases of LIF combined with open PSF, they reported a major complication rate of $20.7 \%$ and overall complication rate of $37.9 \%$. The overall complication rate of $37.5 \%$ in this study is similar to that seen in the literature. However the major complication rate was $12.5 \%$, considerably lower than other reports. Furthermore, all of major complications were infections in our series; therefore no LIF-related major complications occurred.

Limitations of the current study include the lack of a control group and the retrospective nature of the study design. Furthermore, radiographic measurements taken at the inter-stage may be influenced by postoperative back discomfort. However, this study includes data on 151 levels of LIF in 40 patients with adult degenerative scoliosis treated by staged LIF and open PSF. To our knowledge, this study is the largest of its kind reporting on a single institution's data. Furthermore this is the only study that analyzes the relative radiographic contribution of LIF and PSF on staged correction of adult spinal deformity. This study demonstrates that LIF significantly contributed the correction of LL, lumbar coronal Cobb angle and PI-LL mismatch. Lastly, staged LIF and PSF provide excellent clinical and radiographic results and lower complications than seen in prior studies.

\section{CONCLUSION}

LIF combined with staged PSF provides excellent radiographic and clinical outcomes, with reduced perioperative risk in the treatment of adult degenerative scoliosis. LIF significantly contributes the correction in the coronal and sagittal plane in the patients with adult degenerative scoliosis.

\section{CONFLICTS OF INTEREST}

No potential conflict of interest relevant to this article was reported. 


\section{INFORMED CONSENT}

Informed consent was obtained from all individual participants included in this study.

\section{References}

1. Acosta FL, Liu J, Slimack N, Moller D, Fessler R, Koski T : Changes in coronal and sagittal plane alignment following minimally invasive direct lateral interbody fusion for the treatment of degenerative lumbar disease in adults: a radiographic study. J Neurosurg Spine 15 : 92-96, 2011

2. Aharinejad S, Bertagnoli R, Wicke K, Firbas W, Schneider B : Morphometric analysis of vertebrae and intervertebral discs as a basis of disc replacement. Am J Anat 189 : 69-76, 1990

3. Amin BY, Mummaneni PV, Ibrahim T, Zouzias A, Uribe J : Four-level minimally invasive lateral interbody fusion for treatment of degenerative scoliosis. Neurosurg Focus 35(2 Suppl) : Video 10, 2013

4. Anand N, Baron EM, Khandehroo B, Kahwaty S: Long-term 2- to 5-year clinical and functional outcomes of minimally invasive surgery for adult scoliosis. Spine (Phila Pa 1976) 38 : 1566-1575, 2013

5. Anand N, Baron EM, Khandehroo B : Is circumferential minimally invasive surgery effective in the treatment of moderate adult idiopathic scoliosis? Clin Orthop Relat Res 472 : 1762-1768, 2014

6. Baghdadi YM, Larson AN, Dekutoski MB, Cui Q, Sebastian AS, Armitage BM, et al. : Sagittal balance and spinopelvic parameters after lateral lumbar interbody fusion for degenerative scoliosis: a case-control study. Spine (Phila Pa 1976) 39 : E166- E173, 2014

7. Benner B, Ehni G : Degenerative lumbar scoliosis. Spine (Phila Pa 1976) $4: 548-552,1979$

8. Berjano P, Lamartina C : Far lateral approaches (XLIF) in adult scoliosis. Eur Spine J 22 Suppl 2 : S242-S253, 2013

9. Berven SH, Deviren V, Smith JA, Hu SH, Bradford DS : Management of fixed sagittal plane deformity: outcome of combined anterior and posterior surgery. Spine (Phila Pa 1976) 28 : 1710-1715; discussion 1716 , 2003

10. Birknes JK, White AP, Albert TJ, Shaffrey Cl, Harrop JS : Adult degenerative scoliosis: a review. Neurosurgery 63(3 Suppl) : 94-103, 2008

11. Caputo AM, Michael KW, Chapman TM, Jennings JM, Hubbard EW, Isaacs $R E$, et al. : Extreme lateral interbody fusion for the treatment of adult degenerative scoliosis. J Clin Neurosci 20 : 1558-1563, 2013

12. Castro $C$, Oliveira $L$, Amaral $R$, Marchi L, Pimenta $L:$ Is the lateral transpsoas approach feasible for the treatment of adult degenerative scoliosis? Clin Orthop Relat Res 472 : 1776-1783, 2013

13. Dakwar E, Cardona RF, Smith DA, Uribe JS : Early outcomes and safety of the minimally invasive, lateral retroperitoneal transpsoas approach for adult degenerative scoliosis. Neurosurg Focus 28 : E8, 2010

14. Grubb SA, Lipscomb HJ, Coonrad RW : Degenerative adult onset scolio- sis. Spine (Phila Pa 1976) $13: 241-245,1988$

15. Isaacs RE, Hyde J, Goodrich JA, Rodgers WB, Phillips FM : A prospective, nonrandomized, multicenter evaluation of extreme lateral interbody fusion for the treatment of adult degenerative scoliosis: perioperative outcomes and complications. Spine (Phila Pa 1976) 35(26 Suppl) : S322-S330, 2010

16. Johnson RD, Valore A, Villaminar A, Comisso M, Balsano M : Pelvic parameters of sagittal balance in extreme lateral interbody fusion for degenerative lumbar disc disease. J Clin Neurosci 20 : 576-581, 2013

17. Khajavi K, Shen AY : Two-year radiographic and clinical outcomes of a minimally invasive, lateral, transpsoas approach for anterior lumbar interbody fusion in the treatment of adult degenerative scoliosis. Eur Spine J 23 : 1215-1223, 2014

18. Lippman CR, Spence CA, Youssef AS, Cahill DW : Correction of adult scoliosis via a posterior-only approach. Neurosurg Focus 14 : e5, 2003

19. Lonner BS, Murthy SK, Boachie-Adjei 0 : Single-staged double anterior and posterior spinal reconstruction for rigid adult spinal deformity: a report of four cases. Spine J 5 : 104-108, 2005

20. Mundis GM, Akbarnia BA, Phillips FM : Adult deformity correction through minimally invasive lateral approach techniques. Spine (Phila Pa 1976) 35(26 Suppl) : S312-S321, 2010

21. Oskouian RJ Jr, Shaffrey $\mathrm{Cl}$ : Degenerative lumbar scoliosis. Neurosurg Clin N Am 17 : 299-315, vii, 2006

22. Ozgur BM, Aryan HE, Pimenta L, Taylor WR : Extreme lateral interbody fusion (XLIF): a novel surgical technique for anterior lumbar interbody fusion. Spine J 6 : 435-443, 2006

23. Phillips FM, Isaacs RE, Rodgers WB, Khajavi K, Tohmeh AG, Deviren $V$, et al. : Adult degenerative scoliosis treated with XLIF: clinical and radiographical results of a prospective multicenter study with 24-month follow-up. Spine (Phila Pa 1976) 38 : 1853-1861, 2013

24. Rhee JM, Bridwell KH, Lenke LG, Baldus C, Blanke K, Edwards C, et al. : Staged posterior surgery for severe adult spinal deformity. Spine (Phila Pa 1976) 28 : 2116-2121, 2003

25. Shufflebarger HL, Grimm JO, Bui V, Thomson JD : Anterior and posterior spinal fusion. staged versus same-day surgery. Spine (Phila Pa 1976) $16:$ 930-933, 1991

26. Silva FE, Lenke LG : Adult degenerative scoliosis: evaluation and management. Neurosurg Focus 28 : E1, 2010

27. Tempel ZJ, Gandhoke GS, Bonfield CM, Okonkwo DO, Kanter AS : Radiographic and clinical outcomes following combined lateral lumbar interbody fusion and posterior segmental stabilization in patients with adult degenerative scoliosis. Neurosurg Focus 36 : E11, 2014

28. Tormenti MJ, Maserati MB, Bonfield CM, Okonkwo DO, Kanter AS : Complications and radiographic correction in adult scoliosis following combined transpsoas extreme lateral interbody fusion and posterior pedicle screw instrumentation. Neurosurg Focus 28 : E7, 2010

29. Yadla S, Maltenfort MG, Ratliff JK, Harrop JS : Adult scoliosis surgery outcomes: a systematic review. Neurosurg Focus 28 : E3, 2010 